Classification

Physics Abstracts

$05.50-75.10 \mathrm{H}$

\title{
Self-dual anisotropic two-dimensional Ising models with multispin interactions
}

\author{
L. Turban \\ Laboratoire de Physique du Solide (*), Université de Nancy I, CO 140, 54037 Nancy Cedex, France
}

(Reçu le 20 novembre 1981, accepté le 16 février 1982)

Résumé. - On introduit une famille de modèles d'Ising bidimensionnels anisotropes avec interactions à $m$ spins dans une direction et on montre que ces modèles sont self-duaux. Ceci nous permet de localiser la ligne critique lorsque ces modèles présentent une transition de phase unique.

\begin{abstract}
A class of anisotropic two-dimensional Ising models with $m$-spin interactions in one direction is introduced and shown to be self-dual. This enables us to locate the critical line when there is a unique phase transition.
\end{abstract}

In this letter we introduce a class of anisotropic two-dimensional (2D) Ising models with $m$-spin interactions in the spatial (horizontal) direction and the usual two-spin interaction in the temporal (vertical) direction on the rectangular lattice (Fig. 1). These models are self-dual and when unique, a phase transition occurs on the critical line

$$
\sinh \left(2 K_{x}\right) \sinh \left(2 K_{\tau}\right)=1
$$

where $K_{x}$ and $K_{\tau}$ are the couplings (in units of $k_{\mathrm{B}} T$ ) for the spatial and the temporal directions.

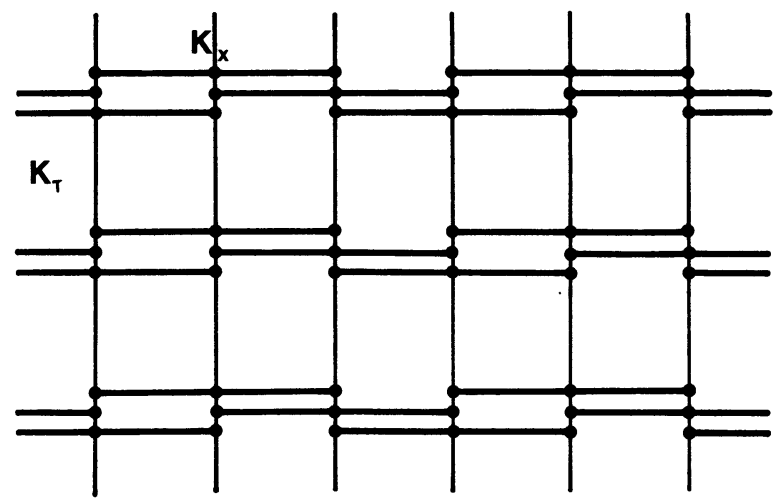

Fig. 1. - Ising model with $m$-spin interactions $K_{x}$ in the spatial direction and two-spin interactions $K_{\tau}$ in the temporal direction $(m=3)$.

(*) Laboratoire associé au CNRS no 155. 
The Hamiltonian of the system is

$$
-\beta \mathcal{H}_{m}=K_{\tau} \sum_{l} s_{i} s_{j}+K_{x} \sum_{L} \prod_{k=1}^{m} s_{k}
$$

where the $\{s\}$ are Ising spins $\left(s_{i}= \pm 1\right)$; the first sum runs over the simple links $l$ joining nearestneighbours in the temporal direction; the second one is over the multiple links $L$ joining $m$ successive spins in the spatial direction. The rectangular lattice has $N$ sites, $N$ simple links $l$ and $N$ multiple links $L\left({ }^{1}\right)$.

The partition function reads :

$$
Z_{m}=\sum_{\{s\}} \mathrm{e}^{-\beta X_{m}}=\sum_{\{s\}} \prod_{l} \exp \left(K_{\tau} s_{i} s_{j}\right) \prod_{L} \exp \left(K_{x} \prod_{k=1}^{m} s_{k}\right) .
$$

Since $\prod_{k} s_{k}= \pm 1$ one may use the identity [1] :

where :

$$
\exp \left(K \prod_{k} s_{k}\right) \equiv \sum_{\lambda=0,1} C_{\lambda}(K)\left(\prod_{k} s_{k}\right)^{\lambda}
$$

$$
C_{0}(K)=\cosh (K) \quad C_{1}(K)=\sinh (K)
$$

to rewrite the partition function as :

$$
Z_{m}=\sum_{\{s\}} \prod_{l} \sum_{\lambda_{\tau}=0,1} C_{\lambda_{\tau}}\left(K_{\tau}\right)\left(s_{i} s_{j}\right)^{\lambda_{\tau}} \prod_{L} \sum_{\lambda_{x}=0,1} C_{\lambda_{x}}\left(K_{x}\right)\left(\prod_{k=1}^{m} s_{k}\right)^{\lambda_{x}} .
$$

Following Savit [1], we now rearrange the spin products in equation (6) so that all factors of $s_{i}$ associated with a given site $i$ are grouped together. Then :

$$
Z_{m}=\sum_{\{\lambda\}} \prod_{l} C_{\lambda_{\tau}}\left(K_{\tau}\right) \prod_{L} C_{\lambda_{x}}\left(K_{x}\right) \sum_{\{s\}} \prod_{i}\left(s_{i}\right)^{\Sigma_{i} \lambda}
$$

where the first sum is over the set of new variables $\lambda_{t}, \lambda_{x}=0,1$. There is one such variable for each link : one $\lambda_{\tau}$ for each $l$ and one $\lambda_{x}$ for each $L . \sum_{i} \lambda$ is the sum over the $\lambda$ variables, two $\lambda_{\tau}$ and $m \lambda_{x}$, associated with site $i$ (Fig. 2). Equation (7) may be rewritten as :

$$
Z_{m}=\sum_{\{\lambda\}} \prod_{i} C_{\lambda_{\tau}}\left(K_{\tau}\right) \prod_{L} C_{\lambda_{x}}\left(K_{x}\right) \prod_{i} 2 \delta_{2}\left(\sum_{i} \lambda\right)
$$

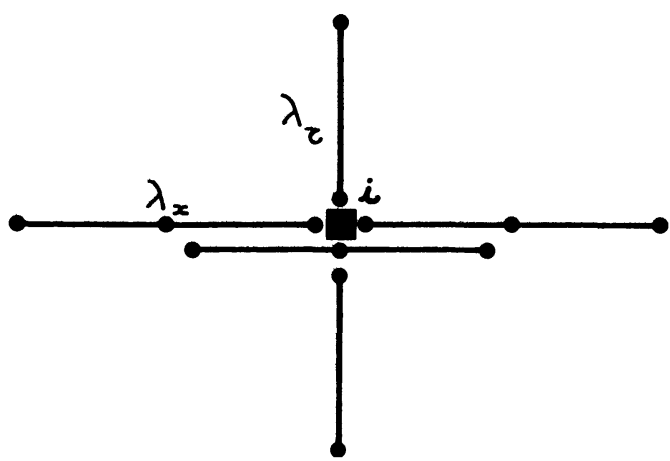

Fig. 2. - The two $\lambda_{\tau}$ and $m \lambda_{x}$ involving site $i$ in the case $m=3$.

( $\left.{ }^{1}\right)$ We take $K_{\mu}>0(\mu=x, \tau)$ without loss of generality since replacing $K_{\mu}$ by $-K_{\mu}$ is equivalent to reversing the spins on some sublattice and leaves $Z_{m}$ unchanged. 
where $\delta_{2}(n)$ is a Kronecker delta function mod. 2, equal to one when $n$ is even and zero when $n$ is odd.

We will now look for a representation of the $\lambda$ variables which automatically satisfies the Kronecker delta functions in equation (8) i.e. such that $\sum_{i} \lambda$ is even. To do this we introduce dual Ising variables $\sigma_{i}= \pm 1$ on the dual lattice (Fig. 3). When $m$ is even the dual variables $\{\sigma\}$

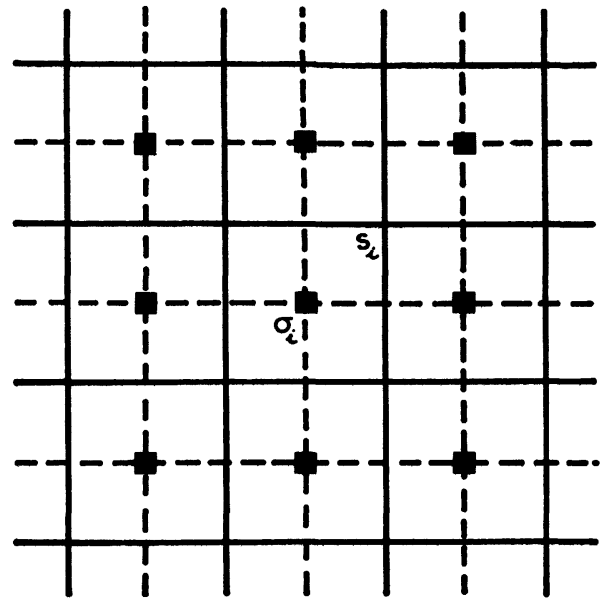

(a)

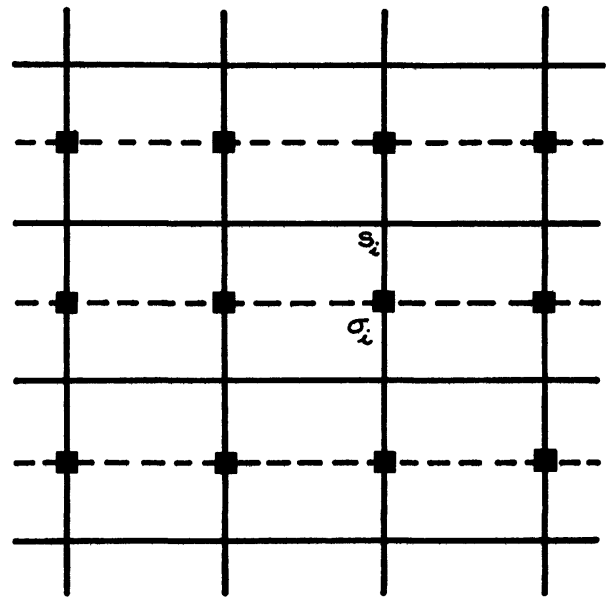

(b)

Fig. 3. - The original (heavy lines) and dual (dashed lines) lattices when $m$ is even (a) or odd (b).
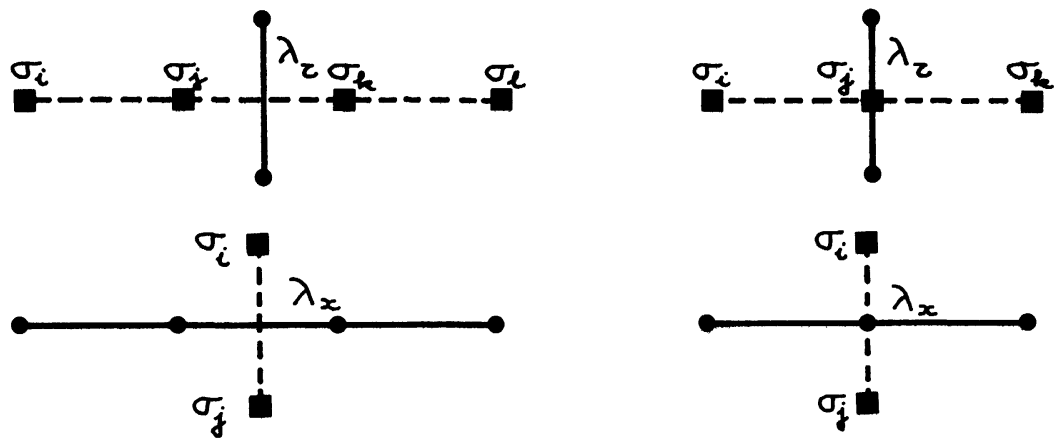

(a)

(b)

Fig. 4. - Correspondence between the $\lambda_{x}$ and $\lambda_{\tau}$ variables on the original lattice (heavy lines) and the spin products on the dual lattice (dashed lines) when $m$ is even $(a)$ or odd $(b)$.

lie on sites situated in the centre of the plaquettes of the original lattice (i.e. the dual lattice is the same as for the usual Ising model), when $m$ is odd they lie in the middle of the temporal links $l$ of the original lattice. There is a one to one correspondence between simple (multiple) links $l(L)$ and their associated variables $\lambda_{\tau}\left(\lambda_{x}\right)$ on the original lattice and multiple (simple) links $L_{\mathrm{d}}\left(l_{\mathrm{d}}\right)$ and their associated dual variables $\prod_{k=1}^{m} \sigma_{k}\left(\sigma_{i} \sigma_{j}\right)$ on the dual lattice (Fig. 4) so that we can write :

$$
\begin{aligned}
& \lambda_{\tau}(\sigma)=1 / 2\left(1-\prod_{k=1}^{m} \sigma_{k}\right) \\
& \lambda_{x}(\sigma)=1 / 2\left(1-\sigma_{i} \sigma_{j}\right) .
\end{aligned}
$$


In this representation, $\sum_{i} \lambda$ is automatically even. For example when $m=3$ we get (Fig. 5) :

$$
\begin{aligned}
\sum_{i} \lambda=5 / 2-1 / 2\left[\sigma_{i} \sigma_{i+\hat{\imath}}+\sigma_{i+\hat{x}} \sigma_{i+\hat{x}+\hat{\imath}}+\right. & \sigma_{i+2 \hat{x}} \sigma_{i+2 \hat{x}+\hat{\imath}}+ \\
& \left.+\sigma_{i} \sigma_{i+\hat{x}} \sigma_{i+2 \hat{x}}+\sigma_{i+\hat{\imath}} \sigma_{i+\hat{x}+\hat{\imath}} \sigma_{i+2 \hat{x}+\hat{\imath}}\right]
\end{aligned}
$$

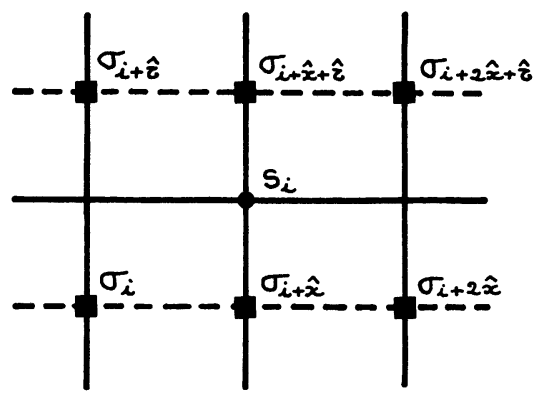

Fig. 5. $-\sum_{i} \lambda$ in the $\sigma$-representation (equations (9) and (10) in the text) when $m=3$. $\hat{x}$ and $\hat{\tau}$ are unit vectors in the spatial and temporal directions.

Each spin enters two terms in the bracket and the sum of spin products may take on the values $5,1,-3$. For any $m$, the possible values of the bracket are

$$
m+2-4 v, \quad v \in N, \quad 0 \leqslant v \leqslant I(m / 2)+1
$$

(where $I(x)$ is the integer part of $x$ ) so that

$$
\sum_{i} \lambda=1 / 2[m+2-(m+2-4 v)]=2 v
$$

is even.

It is clear from equations (9) and (10) that a number of $\{\sigma\}$ configurations lead to the same $\{\lambda\}$ (Fig. 6); in fact this number is equal to the ground-state degeneracy of the model (Fig. 7)

$$
g=\sum_{i=0}^{I(m / 2)} C_{m}^{2 i}=2^{m-1} .
$$

Collecting these results together the partition function may be rewritten as

$$
Z_{m}=2^{1-m} 2^{N} \sum_{\{\sigma\}} \prod_{L_{\mathrm{d}}} C_{\lambda_{\tau}(\sigma)}\left(K_{\tau}\right) \prod_{l_{\mathrm{d}}} C_{\lambda_{x}(\sigma)}\left(K_{x}\right)
$$

Making use of the identity :

$$
C_{\lambda}(K) \equiv \cosh K \exp (\lambda \ln \tanh K)
$$

we get :

$Z_{m}=2^{1-m}\left(2 \cosh K_{\tau} \sinh K_{\tau}\right)^{N / 2}\left(2 \cosh K_{x} \sinh K_{x}\right)^{N / 2} \times$

$$
\times \sum_{\{\sigma\}} \exp \left[\widetilde{K}_{\tau} \sum_{l_{\mathrm{d}}} \sigma_{i} \sigma_{j}+\widetilde{K}_{x} \sum_{L_{\mathrm{d}}} \prod_{k=1}^{m} \sigma_{k}\right]
$$

or :

$$
Z_{m}\left(K_{\tau}, K_{x}\right)=2^{1-m}\left(\sinh 2 \widetilde{K}_{x} \sinh 2 \widetilde{K}_{\tau}\right)^{-N / 2} Z_{m}\left(\widetilde{K}_{\tau}, \widetilde{K}_{x}\right)
$$




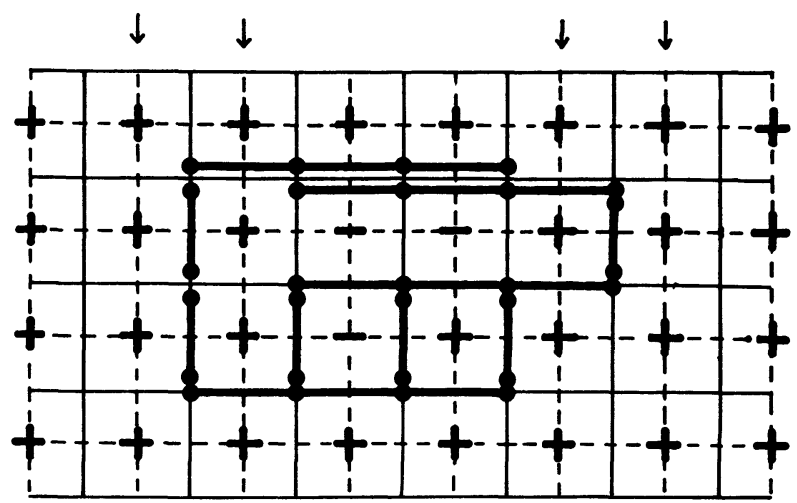

(a)

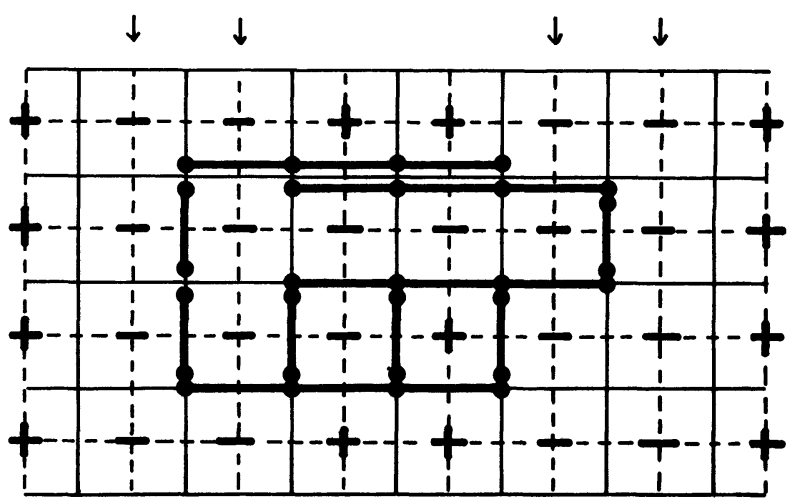

(b)

Fig. 6. - Two configurations $\{\sigma\}$ on the dual lattice (dashed lines) leading to the same $\{\lambda\}$ on the original lattice (heavy lines) for $m=4$. The dual spin configuration on $(b)$ is deduced from $(a)$ by reversing all the spins along the columns indicated by arrows. The Hamiltonian of the system is left invariant under this global symmetry transformation.

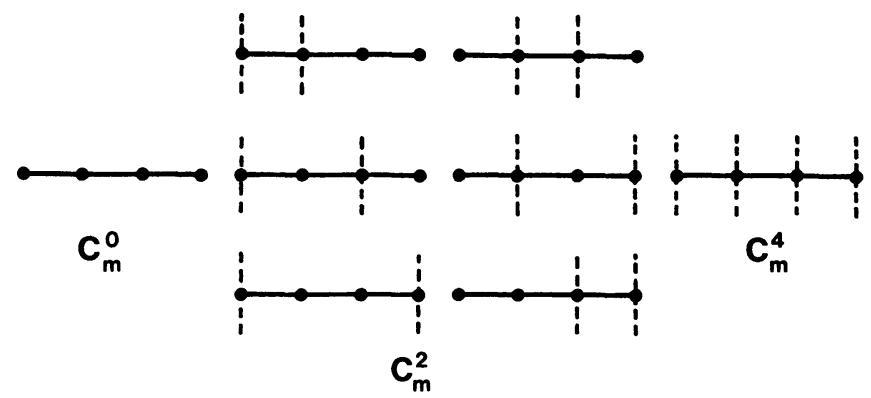

Fig. 7. - The symmetry transformations leaving the 4-spin interaction invariant (reversed spins are indicated by dashed lines). When repeated periodically on the columns of the lattice as in figure 6 , global symmetry transformations are generated. 
where we introduced the dual couplings

or

$$
\widetilde{K}_{\tau}=-1 / 2 \ln \tanh K_{x} ; \quad \tilde{K}_{x}=-1 / 2 \ln \tanh K_{\tau}
$$

$$
\sinh 2 \widetilde{K}_{\tau}=\left(\sinh 2 K_{x}\right)^{-1} ; \quad \sinh 2 \widetilde{K}_{x}=\left(\sinh 2 K_{\tau}\right)^{-1} \text {. }
$$

The free energy per site is

$$
f_{m}\left(K_{\mathfrak{v}}, K_{x}\right)=\lim _{N \rightarrow \infty} \frac{1}{N} \ln Z_{m}\left(K_{\mathfrak{v}}, K_{x}\right)=-1 / 2\left(\ln \sinh 2 \widetilde{K}_{\tau}+\ln \sinh 2 \widetilde{K}_{x}\right)+f_{m}\left(\widetilde{K}_{\mathfrak{v}}, \widetilde{K}_{x}\right)
$$

and the model is self-dual for all $m$. In particular, the line

$$
\sinh 2 K_{x} \sinh 2 K_{\tau}=1
$$

which is invariant under the duality transformation given in equation (20), is the critical line of the model when there is a phase transition and when this transition is unique. Otherwise the duality transformation maps the low temperature region onto the high temperature region of the model.

When $m=1$, we get noninteracting Ising chains in an external field $H=\beta^{-1^{\prime}} K_{x}$. This model has no transition but is known to be self-dual [2]. The model with $m=2$ is the well-known 2D Ising model solved by Onsager [3] which was proved to be self-dual by Kramers and Wannier [4]. When $m=3$ the model bears some similarity with the Baxter-Wu model [5], an Ising model with three-spin interactions on the triangular lattice. Both have the same ground-state degeneracy $g=4$ (Fig. 8) and are related to a model with three-spin interactions on the rectangular lattice with alternate temporal interactions $K_{1}$ and $K_{2}$. Our model is recovered when $K_{1}=K_{2}=K_{\tau}$
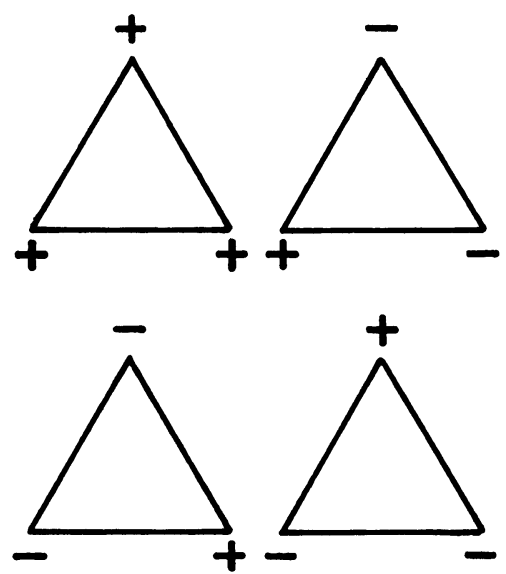

(a)

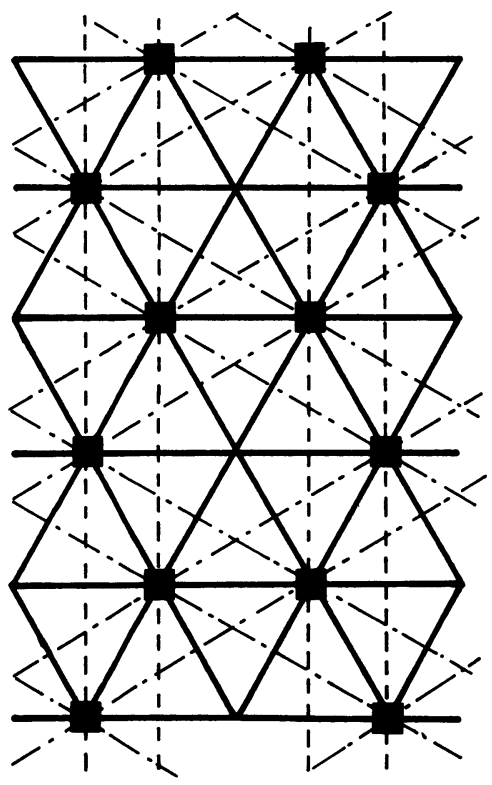

(b)

Fig. 8. - Triangular lattice with 3-spin interactions. (a) The four degenerate ground-states of the elementary interaction. (b) One of the four corresponding global symmetry operations (reversed spins (squares) lie on an hexagonal sublattice). 
and the Baxter-Wu model corresponds to the limit $K_{1} \rightarrow \infty, K_{2} \rightarrow 0$ (Fig. 9). We suspect these models to belong to the same class of universality which might be also that of the 4-state Potts model [6] according to the Den Nijs conjecture [7]. The transition probably becomes first-order above $m=3$ as indicated by mean-field theory on a 1D quantum formulation of the model.

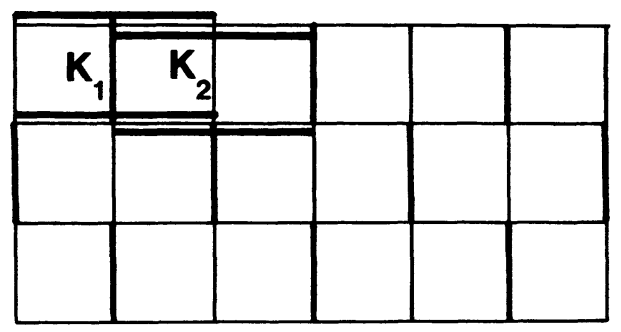

(a)

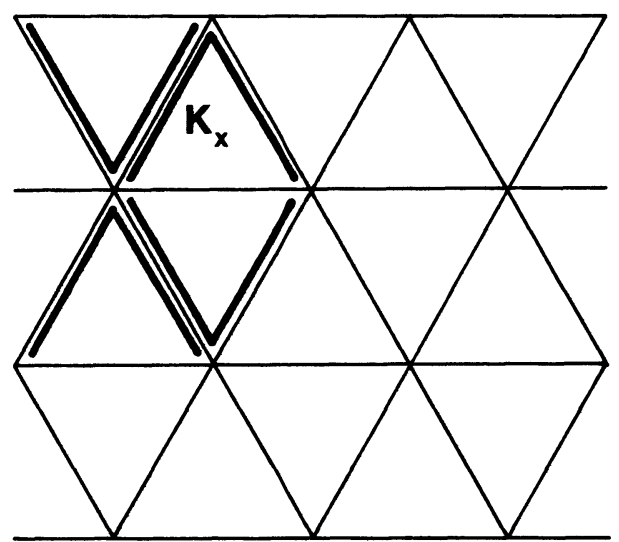

(b)

Fig. 9. - Generalized Ising model with 3-spin interactions $(a)$ and the corresponding Baxter-Wu model (b).

\section{References}

[1] Savit, R., Rev. Mod. Phys. 52 (1980) 453.

[2] Wegner, F. J., J. Math. Phys. 12 (1971) 2259.

[3] ONSAger, L., Phys. Rev. 65 (1944) 117.

[4] Wannier, G. H. and Kramers, H. A., Phys. Rev. 60 (1941) 252.

[5] BaXter, R. J. and Wu, F. Y., Phys. Rev. Lett. 31 (1973) 1294.

[6] Potrs, R. B., Proc. Camb. Philos. Soc. 48 (1952) 106.

[7] Den Nis,, M. P. M., J. Phys. A12 (1979) 1857. 